\title{
A Hybrid Approach for Treating Recurrent Renal Cell Carcinoma (RCC) in a Single Kidney with a Tethered Ureter
}

\author{
Nicole Carter ${ }^{1, \odot}$ Modher Al-Shawi ${ }^{2}$ Peter Royce ${ }^{2,3}$ \\ 1'Department of Radiology, Alfred Hospital, Melbourne, Victoria, \\ Australia \\ 2Department of Urology, Alfred Hospital, Melbourne, Victoria, \\ Australia \\ ${ }^{3}$ Department of Surgery, Alfred Hospital, Melbourne, Victoria, \\ Australia
}

\author{
Andrew O’Brien² Warren Clements ${ }^{1,3, \odot}$
}

J Clin Interv Radiol ISVIR:2021;5:59-61

\begin{abstract}
Keywords

- RCC

- ablation

- ureterolysis

- single kidney

- trauma
\end{abstract}

\author{
Address for correspondence Dr. Nicole Carter, MBBS, Department \\ of Radiology, Alfred Hospital, Commercial Road, Melbourne 2004, \\ Victoria, Australia (e-mail: nicolescarter4@gmail.com).
}

\section{Introduction}

Image-guided ablation (microwave ablation [MWA] or radiofrequency ablation [RFA]) plays an important role in the management of renal tumors. ${ }^{1,2}$ It is a safe, minimally invasive technique that effectively preserves nephron function and carries low rates of recurrence. ${ }^{3-5}$ Ablation is particularly advantageous for patients in whom more traditional surgical management with full or partial nephrectomy confers risks, such as in those with a solitary kidney, limited existing renal function, or complex medical comorbidities. ${ }^{4,5}$

Here, we present a patient with a recurrence of renal cell carcinoma (RCC), following a history of prior partial nephrectomy in her solitary kidney. A decision was made to perform RFA for this patient; however, the presence of scar tissue and altered anatomy due to previous operative management rendered intervention technically challenging. A hybrid approach involving open dissection to facilitate image-guided intervention was utilized.

DOI https://doi.org/ 10.1055/s-0041-1723095 ISSN 2457-0214.

\section{Case Report}

A 69-year-old female initially presented with bacteremia and a CT scan showed an incidental solid RCC at the lower pole of her left kidney, measuring $35 \times 35 \times 33 \mathrm{~mm}$ ( - Fig. 1). Biopsy graded the lesion as Fuhrman nuclear grade 2 and CT staged the lesion pT1a NO MO. This was in the setting of a solitary kidney due to prior total right nephrectomy performed, following traumatic motor vehicle injury 40 years prior, with baseline creatinine 70 to 90 micromol/L. The patient's complex medical history included obesity (body mass index [BMI] of 36), hypertension, a 45 pack-year smoking history, and a right leg at-knee amputation.

An open partial nephrectomy was subsequently performed to resect the tumor, with no residual mass seen on initial 6-week postoperative imaging ( Fig. 2). However, surveillance CT 6 months later demonstrated a $23 \times$ $26 \times 22 \mathrm{~mm}$ ovoid recurrence at the margin of the surgical site, and at the medial aspect of the collecting system ( - Fig. 3a).The 


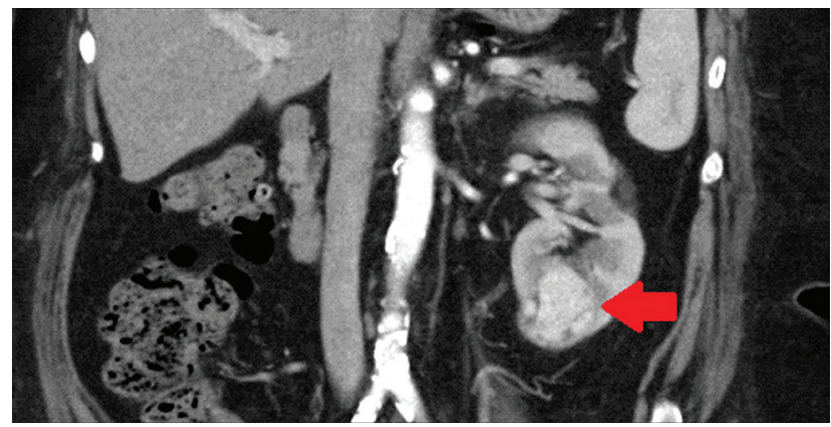

Fig. 1 Arterial phase CT with coronal reformats showed a $35 \times 35 \times$ $33 \mathrm{~mm}$ solid mass at the lower pole of the solitary left kidney (arrow). Biopsy confirmed the imaging diagnosis of renal cell carcinoma.

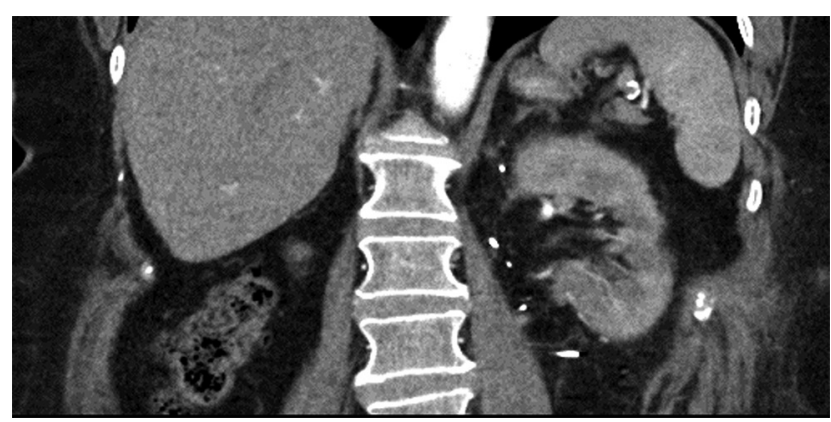

Fig. 2 Arterial phase CT with coronal reformats after partial nephrectomy showed a clear margin.

ureter was tethered to the lesion at the pelviureteric junction ( - Fig. 3b), likely due to surgical scar tissue.

The patient and imaging were discussed at a multidisciplinary meeting and a combined approach with open exploration, ureterolysis, and intraoperative ultrasound-guided ablation of the lesion was agreed upon.

Under general anesthesia, a rigid cystoscopy and retrograde pyelogram confirmed a tethered upper ureter, into which a 6-French ureteric stent was passed under image intensifier guidance over a guidewire. The patient was moved from lithotomy to left lateral position, and an incision was made over the left flank above the 12th rib, near the previous partial nephrectomy incision. Dissection and initial mobilization were difficult as anticipated, due to scarring from the previous open partial nephrectomy. Once renal exposure was achieved, the tethered area of the kidney and ureter was carefully dissected without injury to the ureter, with excellent exposure of the recurrent lower pole tumor. Needle core biopsy was obtained for histology before proceeding with tumor ablation. Histology later confirmed RCC recurrence. A curved $9 \mathrm{MHz}$ ultrasound probe (GE Healthcare) was placed onto the exposed renal capsule; thereafter, a single-lead RFA probe (Cool-tip $3.5 \mathrm{~cm}$, Covidien) was placed into the lesion under ultrasound guidance (-Fig. 4) and a 12-minute ablation cycle performed. As the kidney was mobilized from the perinephric fat, it was able to be manipulated to ensure placement of the single needle parallel to the long axis of the ovoid-shaped recurrence. Gelfoam was placed prior to closure to separate the
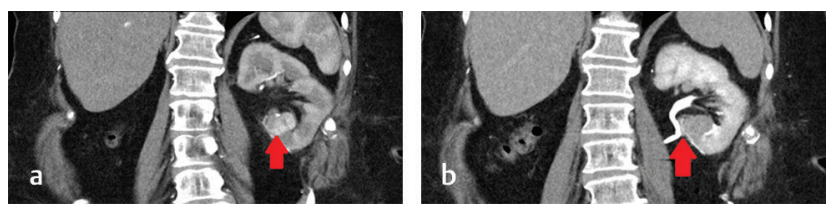

Fig. 3 (a) Arterial phase CT with coronal reformats at 6 -month surveillance a demonstrated $23 \times 26 \times 22 \mathrm{~mm}$ focus of tumor recurrence at the medial margin of the kidney (arrow). (b) Delayed phase CT with coronal reformats shows the ureter deviated laterally (arrow), directly in contact with the partial nephrectomy site. Contrast washout also noted within the adjacent tumor.

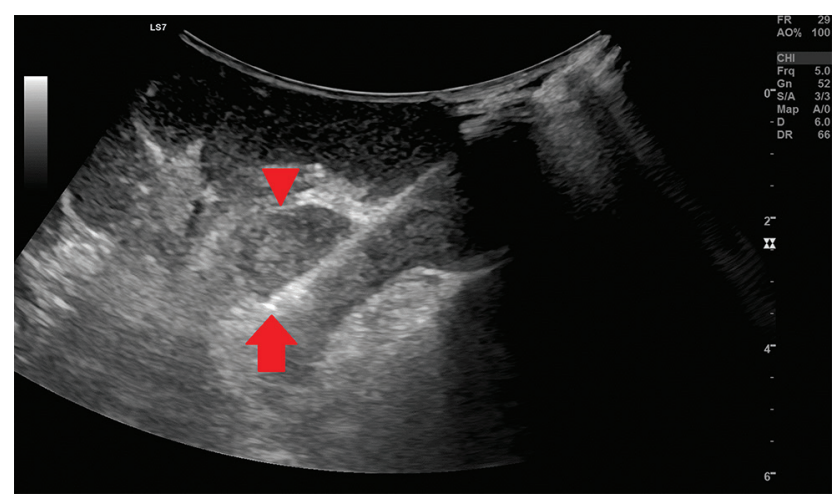

Fig. 4 Intraoperative ultrasound directly onto the renal capsule shows the ablation probe placed (arrow) within the mass (arrowhead).

ablated renal tumor from the stented ureter and provide additional thermal buffer.

The procedure was well-tolerated by the patient with nil operative complications. She was able to pass urine adequately following the procedure with no hematuria and was discharged home 3 days later with stable renal function. The ureteric stent was removed after 4 weeks, and CT performed at 6 months ( - Fig. 5a) and 13 months ( - Fig. 5b) demonstrated conserved volume of normal renal parenchyma, shrinking tumor scar, and no tumor enhancement. Renal function has remained stable at baseline, and there has been no requirement for postoperative dialysis.

\section{Discussion}

Traditionally, the mainstay treatment approach to RCC management has been total nephrectomy of the affected kidney. ${ }^{1,3}$ However, the more widespread use of high-resolution diagnostic imaging has led to the more frequent diagnosis of smaller lesions in asymptomatic patients, and also led to the development of nephron-sparing techniques. The current available treatment options for small RCC include active surveillance, percutaneous ablation, radiation therapy, and surgical resection. ${ }^{3}$ RFA, MWA and cryotherapy ablation are increasingly useful adjuncts in the management of those who are poor surgical candidates due to advanced age or comorbidities, or who have limited existing functional renal reserve on account of single kidney or prior partial resection. The minimally invasive approach allows for less perioperative risk, shorter hospital stays, better preservation of renal 


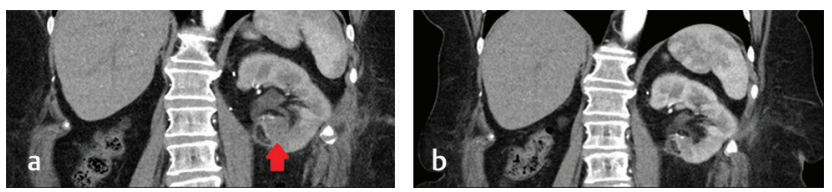

Fig. 5 (a) Arterial phase CT with coronal reformats 6 months after hybrid treatment showed no residual enhancement and mild shrinkage of the tumor scar (arrow). (b) Portal venous phase CT with coronal reformats 13 months after hybrid treatment showed significant shrinkage of the tumor scar (arrow).

function, and low-incidence of major complications. ${ }^{4}$ While there are no randomized controlled trials comparing ablation with surgery for RCC, multiple studies have reported excellent disease control following RFA. ${ }^{5-8}$ A 2018 review of 11 consecutive patients with local RCC recurrence treated with thermal ablation demonstrated significant efficacy with regard to technical success, local response, and lowrate of disease progression. ${ }^{9}$ Studies have shown equivalency between RFA, MWA and cryotherapy with regard to therapeutic outcomes, preservation of renal function and adverse event rates. ${ }^{9,10}$ The choice of method may depend on institutional access, experience and preference.

Treatment options considered in this patient included a percutaneous approach combined with pyeloperfusion by saline infiltration, either through nephrostomy or a ureteral catheter. This was deemed logistically difficult, and it was judged that damage to the collecting system was still likely. It was ultimately decided to take the treatment path shown, where stenting and ureterolysis could then free the ureter from its close adherence to the lesion, allowing space for intraoperative RFA to be safely performed. Cryoablation was not considered as the ablative method, due to limited access to cryotherapy in the authors' country. Ultrasound was still used intraoperatively, as the lesion was at the medial margin of the kidney, which allowed the outer cortex to remain preserved.

The patient's baseline creatinine was at the upper limits of normal in the context of a prior total right nephrectomy and partial left nephrectomy. Maintaining an approach which was nephron-sparing, causing appropriate tumor death, and also providing a small margin was a fine balance, which was successfully achieved due to the approach used. The other balance which needed to be considered was how to protect the ureter from thermal injury to allow adequate ablation to be performed. Ureteric injury or delayed thermal stricture would place the patient at risk of future port-renal obstruction and would almost certainly precipitate the need for lifelong dialysis. In our patient, in addition to the ureteric tethering to the mass, a simple percutaneous approach was also deemed challenging due to body habitus with central abdominal girth, limiting placement of the long ablation probe within the small CT gantry. The recurrent lesion was also not visible on percutaneous ultrasound. Conversely, a purely open resection of the tumor would be technically challenging due to existing adhesions from the previous operation, which could potentially result in an unacceptable level of nephron loss and similar risk of renal failure.

\section{Conclusion}

This case shows a successful approach to treating RCC recurrence in a single kidney, where the ureter was adherent to the mass from existing scar tissue. Using radiofrequency ablation after open surgical dissection and ureterolysis allowed for adequate ablation to be performed; in this patient, it achieved a fine balance of tumor death and a small margin, avoided ureteric injury, and thus prevented the need for postoperative dialysis.

\section{Funding}

None.

\section{Conflicts of Interest}

The authors declare no conflicts of interest.

\section{References}

1 Cohen HT, McGovern FJ. Renal-cell carcinoma. N Engl J Med 2005;353(23):2477-2490

2 Escudier B, Porta C, Schmidinger M, et al; ESMO Guidelines Committee. Electronic address:, clinicalguidelines@esmo. org. Renal cell carcinoma: ESMO Clinical Practice Guidelines for diagnosis, treatment and follow-up. Ann Oncol 2019; 30(5):706-720

3 Johnson BA, Sorokin I, Cadeddu JA. Ten-year outcomes of renal tumor radio frequency ablation. J Urol 2019;201(2):251-258

4 Uhlig J, Kokabi N, Xing M, Kim HS. Ablation versus resection for stage $1 \mathrm{~A}$ renal cell carcinoma: national variation in clinical management and selected outcomes. Radiology 2018; 288(3):889-897

5 Cooper CJ, Teleb M, Dwivedi A, et al. Comparative outcome of computed tomography guided percutaneous radiofrequency ablation, partial nephrectomy or radical nephrectomy in the treatment of stage T1 renal cell carcinoma. Rare Tumors 2015;7(1):5583

6 Gervais DA, McGovern FJ, Arellano RS, McDougal WS, Mueller PR. Radiofrequency ablation of renal cell carcinoma: part 1, Indications, results, and role in patient management over a 6-year period and ablation of 100 tumors. AJR Am J Roentgenol 2005;185(1):64-71

7 MacLennan S, Imamura M, Lapitan MC, et al; UCAN Systematic Review Reference Group; EAU Renal Cancer Guideline Panel. Systematic review of oncological outcomes following surgical management of localised renal cancer. Eur Urol 2012;61(5):972-993

8 Psutka SP, Feldman AS, McDougal WS, McGovern FJ, Mueller P, Gervais DA. Long-term oncologic outcomes after radiofrequency ablation for $\mathrm{T} 1$ renal cell carcinoma. Eur Urol 2013;63(3):486-492

9 Zhou W, Herwald SE, McCarthy C, Uppot RN, Arellano RS. Radiofrequency ablation, cryoablation and microwave ablation for T1a renal cell carcinoma: A comparative evaluation of therapeutic and renal function outcomes. J Vasc Interv Radiol 2019;30(7):1035-1042

10 Martin J, Athreya S. Meta-analysis of cryoablation versus microwave ablation for small renal masses: is there a difference in outcome. ? Diagn Interv Radiol 2013;19(6):501-507 